\title{
Metallographic Preparation of Incoloy 800 Tube Material for EBSD
}

\begin{abstract}
A.J. Lockley
Canadian Nuclear Laboratories, Chalk River Laboratories, Chalk River, Ontario, Canada, K0J 1J0
\end{abstract}

Incoloy 800 is a nickel-iron-chrome alloy used where high temperature- and corrosion -resistance is required with typical configuration and application being tube material for heat-exchangers. During manufacturing, the boilers tubes are subject to cold-work introducing residual strain into critical regions of the tube. In characterizing the impact of this cold-work on the microstructure, tube material was prepared using metallography for examination by Electron Backscatter Diffraction (EBSD). One challenge associated with sample preparation for this application can be attributed to the the wall thickness of the tube, being only 1 millimeter (Figure 1). Sectioning, mounting and polishing of the sample may introduce additional strain, and inflate results obtained by subsequent EBSD. Most such artifacts can be circumvented by electro-polishing, however; this approach brings a different set of complications: electronic differences from inclusions leaving large pits, and heavy rounding of sample edges (an area of specific interest). Standard metallographic mounting methods introduce other complications as well. Modifications to sample preparation, specifically mounting and the final polish, were found to mitigate these complications.

To help maintain edge retention several parallel specimens were cut and assembled in a high-conductive silver epoxy to bind and surround the specimens. The primary benefit of the silver epoxy is that its conductive properties encasing the specimens acts to minimize charging and drift in the scanning electorn microscope (SEM) and improve overall EBSD quality. The assembly in silver epoxy was supplemented with an epoxy casting resin to complete the mount to a standard $30 \mathrm{~mm}$ diameter metallographic mount configuration (Figure 2). A compression mounting material was not used because it nominally requires compressive loading, from a hydraulic cylinder, to be applied to both the mounting material and the specimen as part of the forming process. Previous laboratory experience has shown that delicate material, such as small springs or the thin walls of tube specimens, may be subject to deformation during this type of mounting process. Therefore, this method was avoided to minimize the introduction of strain resulting from any such deformation.

The mounted specimens were prepared using standard metallographic methods, however; the final polish employed a chemical-attack polish comprising cooper (II) chloride, hydrochloric acid and colloidal silica [1] that removed surface damage induced by previous polishing steps. Optical examination using an inverted metallograph was used to inspect the effectiveness of the attack-polish. A prepared surface should appear featureless but structure and surface details, including preparation damage such a light scratching, can be observed by defocusing the microscope and viewing using a small aperature (Figure 3). Such observable damage should be eliminated by further polishing prior to EBSD analysis. 
This preparation method has been proven successful in providing strain orientation maps with minimal preparation damage and gives the additional benefit of edge retention to enable strain detail at the surfaces (Figure 4).

\section{References}

[1] G. Petzow, Metallographic Etching, $2^{\text {nd }}$ Ed., ASM international, 1999.

[2] EBSD images provided by C. Mayhew, Surface Science Lab, CNL, Canada.

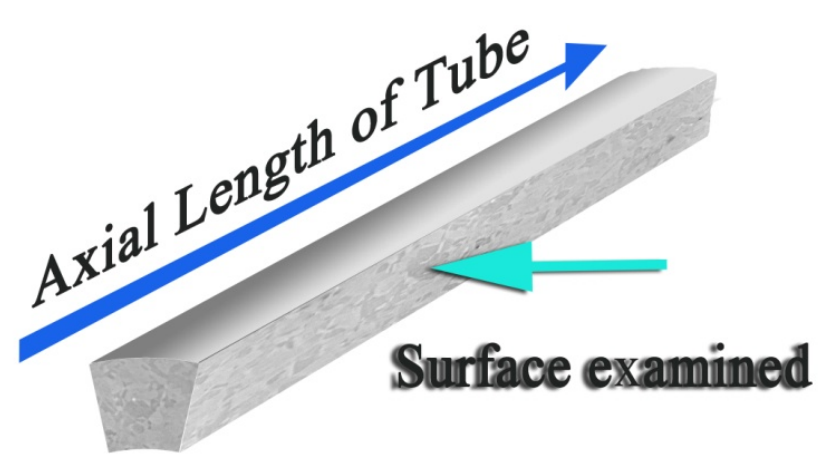

Figure 1 Schematic of a single section of a tube wall specimen.

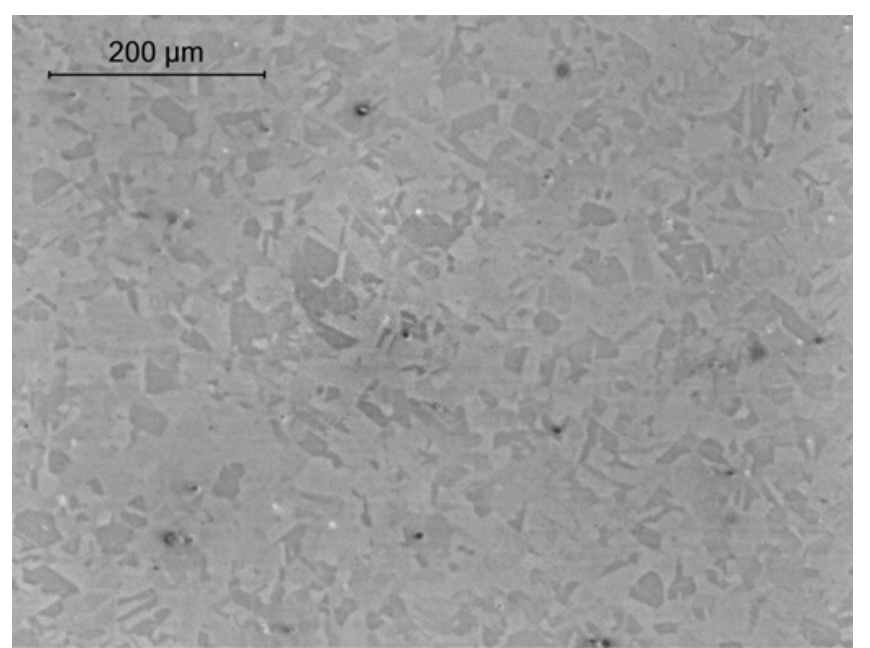

Figure 3 Optical micrograph of Incoloy 800 microstructure. Image is slightly de-focused to reveal detail.

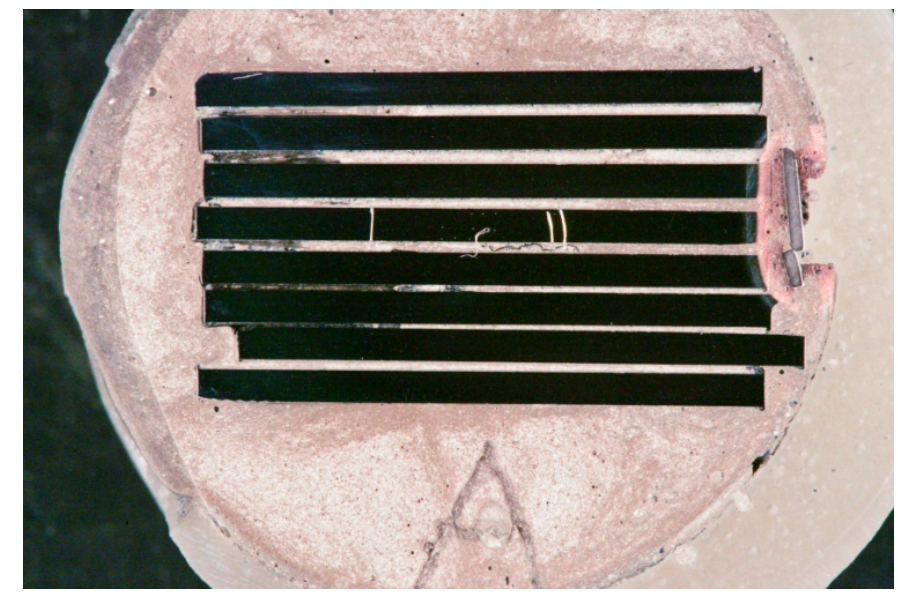

Figure 2 Photograph of a metallographic mount containing tube cross-sections.

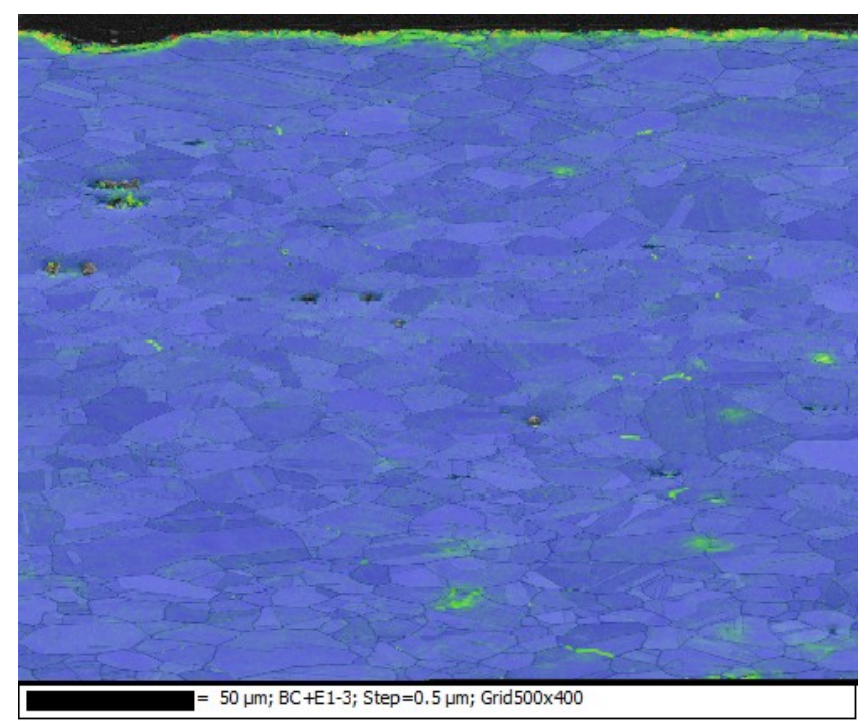

Figure 4 Strain-orientation map near the outside surface of the tube (top of image). 